\title{
Particle therapy masterclass
}

\author{
Panagiota Foka ${ }^{1 *}$, Aristeidis Mamaras ${ }^{2}$, Damir $\mathrm{Skrjiel}^{3}$, Joao $\mathrm{Seco}^{4}$, Christian Graeff ${ }^{5}$, Marco Pulia ${ }^{6}$, Hans-Peter Wieser $^{7}$ and \\ Niklas Wahl ${ }^{4}$ \\ ${ }^{1}$ GSI Helmholtzzentrum für Schwerionenforschung, Planckstraße 1, 64291 Darmstadt, Germany \\ ${ }^{2}$ AUTH University Campus, Administration Building, 54124, Thessaloniki, Greece \\ ${ }^{3}$ UNSA Obala Kulina bana 7/II, Sarajevo 71000, Boznia and Herzegovina \\ ${ }^{4}$ DKFZ Im Neuenheimer Feld 280, 69120 Heidelberg, Germany \\ ${ }^{5}$ Department of Electrical Engineering and Information Technology, Technical University, Darmstadt, Germany \\ ${ }^{6} \mathrm{CNAO} 642 \mathrm{~W}+92$ Pavia, Provincia di Pavia, Italy \\ ${ }^{7}$ LMU Geschwister-Scholl-Platz 1, 80539 München, Germany
}

\begin{abstract}
The aim of the new Particle Therapy MasterClass (PTMC) was to develop an educational and training environment in which anyone can learn about fundamental and applied research in particle therapy. The PTMC was recently integrated into the International MasterClass 2021 online programme that attracted 1500 students from 37 institutes in 20 countries, worldwide. The PTMC focuses on the topic of cancer treatment, a particularly sensitive and socially relevant topic. The main idea is to (a) provide a basic understanding of cancer radiation therapy, (b) demonstrate that fundamental properties of particle interactions with matter, which are used for detection in physics experiments, are also the basis for treating cancer tumours; and (c) show that the same accelerator technologies are used in both, research laboratories and therapy centres. For the hands-on session, the open-source professional treatment planning software matRad is used, developed for research and training by the German Cancer Research Center - DKFZ. Ultimately, students are shown "what physics has to do with medicine" and what are the various possibilities that physics and STEM studies may open up for job opportunities in fields that are lacking expert personnel.
\end{abstract}

\section{Introduction}

The new Particle Therapy MasterClass, PTMC, developed in 2019 and presented in this paper, aims at highlighting applications of fundamental research for the benefit of society. While the mission and mandate of research institutes, such as CERN, GSI and others, is fundamental research, the developed technologies and acquired knowledge find applications in many different domains. The aim of the PTMC is to enhance awareness of such contributions focusing on the particularly sensitive societal topic of cancer therapy, while also enhancing awareness on advanced possibilities of cancer tumour treatment using particles such as protons and heavier ions.

The concept of the PTMC combines different pedagogical elements (Section 2) giving the opportunity to participants to become scientists for a day. Elaborating on the theme "from particle physics to particle therapy" it was initially developed targeting high-school students within the International MasterClasses, IMC [1], the flagship project of the International Particle Physics Outreach Group IPPOG [2]. Additionally, it can be easily tailored to different needs and education levels. The developed concept and available material via the PTMC web page [3] were already used to complement training of university students, as basis for a full week specialised school and for events such as the CERN Open Days and the Science Days of Montenegro.

Within the IMC project, masterclasses are usually organised as full day events, with a typical agenda, followed by the participating institutes. During 6 weeks before the Easter vacations, 3-5 institutes participate every day, inviting highschool students to immerse in the world of particle physics giving them a taste on "what scientists do". Adapting to an online mode due to the Covid pandemic, students were invited by the institutes to join a zoom session, instead of being invited at the institutes in person. In several cases, this was announced broadly and students from the whole country could join the online sessions. The PTMC specifically shows to students "what physics has to do with medicine", and triggers questions on how particles can be used for cancer therapy.

The PTMC, coordinated by GSI, was included for the first time in the IMC2020 schedule after a first local test at GSI and a pilot one, in early 2019, with the participation of GSI [4], CERN [5] and DKFZ [6] Heidelberg. This core team represents the world leading research institutes in their domains that also provided tangible applications for health: GSI treated the first 450 patients in Europe with carbon ions, CERN hosted the Proton-Ion Medical Machine Study (PIMMS) [7] design team. DKFZ is Germany's national cancer research institute, which is also closely connected to the HIT Heidelberg ion therapy facility [8]. The first PTMC session in IMC2020 was performed in Mexico [9], attracting about

\footnotetext{
* Corresponding author: yiota.foka@,cern.ch
} 
200 students, while the rest of the sessions had to be cancelled. In the IMC2021 season, the PTMC sessions were performed online, and with no limitation on registrations, they attracted more than 1500 students around the world. During the 6 PTMC sessions a total of 37 institutes from 20 countries participated, with about 6 institutes performing the PTMC in the same day.

\section{Pedagogical elements}

The PTMC agenda integrated and expanded the basic elements of the IMC that include, in the morning, introductory lectures and a visit to an experiment or lab, while in the afternoon, a hands-on session, followed by a common videoconference among all participating institutes. Each PTMC online session started with a video, showing a particle therapy procedure in a virtual therapy centre to trigger curiosity and interest. Further, it provided an overall visual impression, so that students could relate the specific presentations that followed to the broader image and procedures. The lectures covered a wide range of topics, supported by videos to keep the interest of students. Introductory lectures covered the basics of cancer biology and treatment options, the physics and biology of exposure to ionizing radiation, imaging devices and detectors, as well as accelerators used for cancer diagnosis and treatment. Due to the multidisciplinary nature of the topic, the introductory lectures can be tuned to or complemented by lectures on the expertise of the different institutes. This includes, for example, fundamental detector or software developments that are used in medicine for imaging, diagnostics, dosimetry, as they are critical parts of the treatment procedures where the impact of breakthrough developments on detectors for physics experiments is clear. The importance of computing and software developments is also highlighted including machine learning techniques and their possible applications in these fields. The message is always that: technologies developed for fundamental research find applications for medicine.

Highlighting the use of accelerators for society, students were surprised to learn that from about 30000 accelerators that are in operation world-wide, about $6 \%$ is used for research, while $1 / 3$ is used for medicine and the rest for different industrial applications. Adapting technologies and methods developed for fundamental particle physics research, sophisticated medical accelerators can deliver particle beams at a desired depth targeting a tumour. Students are also shown that fundamental properties of particle interactions with matter, which are used to detect them in physics experiments, are also the basis for treating cancer tumours. Figure 1a shows, as an example, the Bethe Bloch energy loss used for particle identification in the TPC detector of the ALICE [10] experiment at the CERN LHC, while the 1b plot presents the energy deposition as function of depth in water.
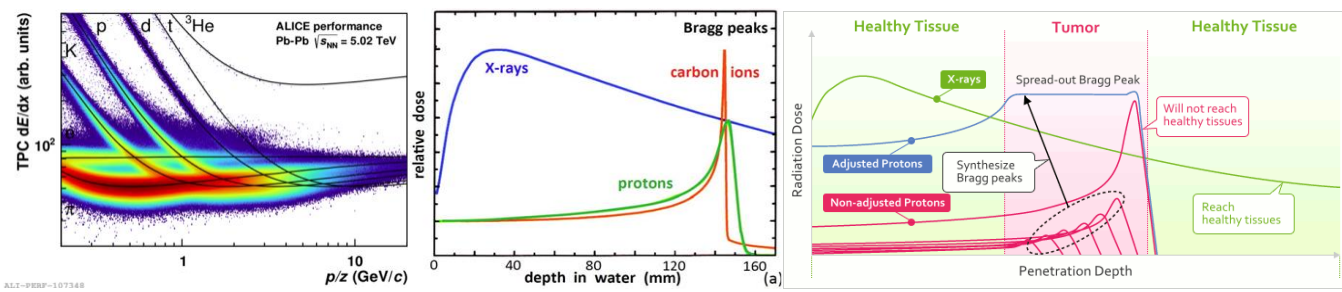

Figure 1: Bethe Bloch energy loss used for particle identification (a); energy deposition as function of depth for photons, protons and carbon ions used for Hadron Therapy (b); and spread-out Bragg peak (c).

Here, protons and carbon ions reveal their dose deposition characteristics, manifesting in the Bragg peak, which is the basic motivation for using "heavy" charged particles radiation therapy. One can clearly observe that photons lose most of their energy entering the tissue and they still deposit energy in healthy tissues behind the tumour while ions deposit most of their energy at the end of their trajectory before they come to a full stop. By tuning the energy of the accelerator and adapting the number of ions that are being accelerated, one can scan the entire tumour homogeneously, what is known as spread-out Bragg peak (SOBP), shown on the last plot.

During the initial pilots in 2019 visits to HIT, CERN and GSI were possible. Since 2020 online visits were offered, due to the pandemic, at the ALICE heavy-ion experiment. They were complemented with online visits during the afternoon common video-conferencing at the GSI experimental room where carbon ion therapy was pioneered in Europe, and in the CNAO [11] ion therapy centre, close to Milano (Figure 2). Experts from these institutes provided an excellent interactive help, discussing with the students the results. These online visits further highlighted the connection between heavy-ion physics and heavy-ion therapy.

In general, the PTMC highlighted that technology and knowledge transfer "from physics to clinics" resulted in the four carbon-ion therapy centres in Europe. Following the PIMMS initiative, accelerator experts of the CERN NIMMS group work currently on the design of components for a next generation ion therapy machine adapting hi-tech technologies for med-ical accelerators. As an example, Figure 3 shows the accelerator layout and architect's concepts (courtesy "Kaprinis architects") for a heavy-ion therapy facility which is proposed to be built in South East Europe (SEE). The PTMC was performed by several SEE institutes that provided material in several local languages, supporting capacity building in the area. 

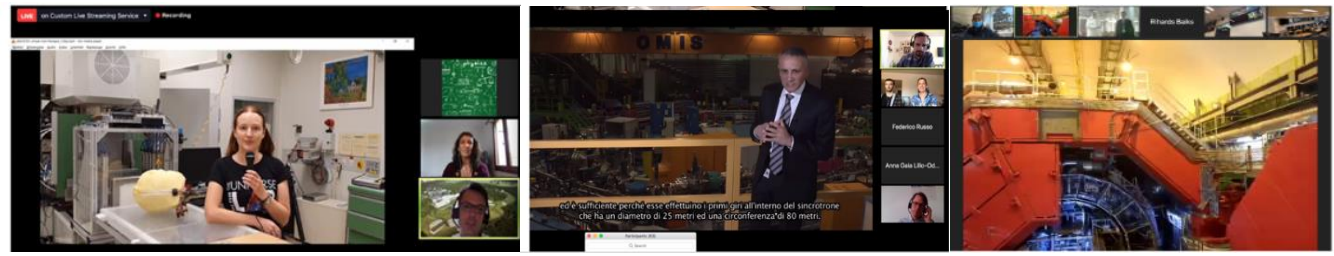

Figure 2: Online visits at GSI heavy-ion research centre, CNAO ion therapy centre and the ALICE heavy-ion experiment.
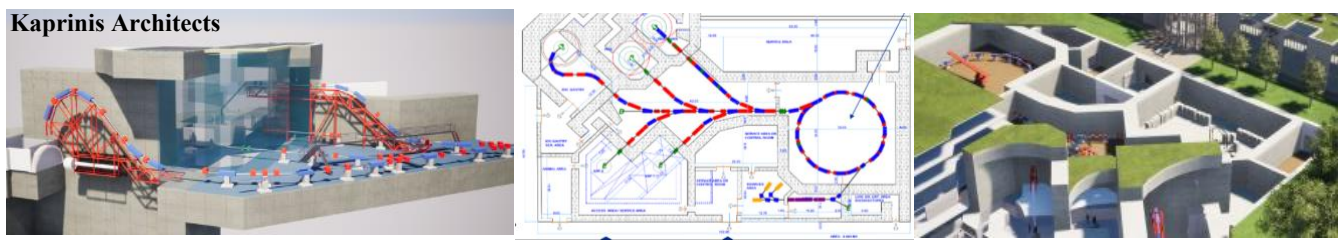

Figure 3: Accelerator layout of a next generation ion medical facility.

Before the PTMC hands-on session, the students were introduced to the concepts of dose calculation, dose optimization and radiotherapy treatment plans. In particular, school students can learn here how elemental mathematical concepts (e.g., learned from calculus) are required and applied to solve actual technical problems. The PTMC hands-on session itself is based on the open-source treatment planning toolkit developed by DKFZ for research and training (Figure 4) $[12,13,14]$. It is used to simulate and optimize the therapeutic intensity-modulated dose distribution that the accelerating structures have to deliver to the tumour using photons, protons or carbon ions according to "prescriptions" of the students who can appreciate the differences of these radiation modalities and use them optimally according to different cases. While matRad's computations were validated with commercial treatment planning software actually used for therapies, it is not licensed for treatment itself but uses a GPLv3 open-source license. This facilitates matRad's lightweight, flexible, accessible and performant code, useful for research and such educational applications as the PTMC. Further, depending on the level of the student, special educational versions can be provided with reduced treatment planning complexity of the GUI.

The provided data are a TG-119 phantom and anonymous computer tomography (CT) scans of head, liver and prostate tumour cases from the CORT dataset [15]. MatRad includes a graphical user interface (GUI) resembling typical commercial systems to present the data in $3 \mathrm{D}$, provides possibilities to rotate interactively and presents slices in $2 \mathrm{D}$ projections. The GUI includes graphical tools to visualize the beam directions and volumes to be irradiated.

For the dose optimisation, one can use multiple objectives and constraints for dose prescription, e.g., define a minimum and/or maximum dose value to be achieved within a structure or organ [14]. The visual result on the display, based on a colour scale, is very intuitive to comprehend and easy to associate with the resulting histograms of the delivered dose to the target tumour and organs at risk to avoid. Students can select different angles for irradiation to minimize the deposited dose in each trajectory while accumulating the required dose in the target volume. They can witness the different characteristics of protons and carbon ions, that, in contrast to photons, deposit very little energy in the tissues before and practically none after the tumour (Figure 4).

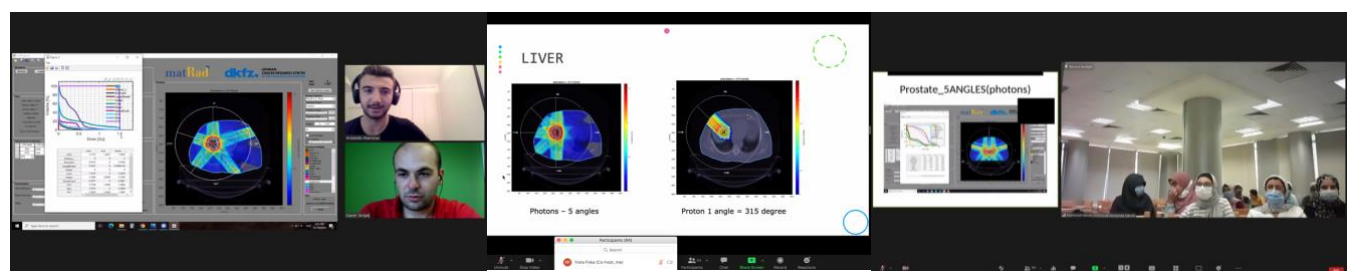

Figure 4: matRad treatment planning application.

The hands-on session is typically divided into three sections. The first section aims at highlighting the difference of photon and charged particle irradation on the TG-119 phantom, showing, for example, that many beams are required with photons to cover the tumour while the sparing of healthy tissue can be challenging, whereas protons achieve at least similar results with already one or two beams.

The second part introduces carbon ions and radio-biological effectiveness on a patient case. The institutes and/or students can choose between the liver and head-and-neck case for this exercise. This introduces versatility to the later videoconference, where students present and exchange their findings.

The last part critically introduces the "downside" of charged particle therapy, that is, its sensitivity to uncertainties that might change the net range in the patient and distort the planned dose distribution when the patient is, e.g., misaligned.

Instructions for installing matRad specific software, prepared by the DKFZ colleagues, are available via the PTMC web pages, including recordings and are sent to participants in advance, although installation can be handled even during 
the session. In order to support the PTMC local organising teams of each participating institute, and prepare their tutors and moderators, weekly dedicated tutorials were offered during the IMC2021 season but also training sessions at request. The PTMC web pages [3] provide a large variety of material in several languages, including links to the "PTMC in a kit" google drive which contains the necessary material needed to perform the PTMC, including recordings with instructions. In addition, it links to the indico agendas of institutes that performed already the PTMC, including their presentations in their local languages.

\section{PTMC tutorial}

To enable researchers to become tutors for the PTMC, tutorials are offered as training. These tutorials focus mainly on the hands-on session with matRad to familiarize with the matRad GUI and provide a deeper understanding of the underlying cancer treatment planning concepts.

The tutorials explain the possibilities of the software that is accessible through the GUI (Figure 5), which includes four different tabs: The first "workflow" tab controls the execution of the major steps of the treatment planning process. Thus, it contains buttons for loading the provided patient data as well as DICOM files, data from binary files and already prescribed doses. The "dose calculation" button allows to either compute the dose influence matrix to be used for a subsequent dose optimization of a new treatment plan or recalculate the dose for a different patient geometry (e.g., a shifted patient). The "optimization" button triggers dose optimization of the beam fluence, i.e., the beamlet (named "bixel" in matRad) weights, that yield the best possible dose distribution according to the clinical objectives and constraints underlying the radiation treatment. For photons, a beamlet/bixel is a small photon intensity element used to illustrate a portion of an intensity-modulated radiotherapy (IMRT) beam for the optimization of the intensity distribution or for dose calculations for the treatment planning [16], while for charged particles it refers to an individual spot with distinct lateral position and energy, forming one of the localized Bragg-peaks that compose SOBP.

Next, is the "plan" tab, where the parameters of the plan are inserted. In this tab, the students can input the beamlet/bixel width (or spot spacing for particles), the gantry and couch angles, the type of the radiation method, the number of fractions of the prescribed dose as well as the "isocenter" (the reference point in space around which, the gantry and couch rotate) coordinates.

The plan tab is followed by the "objectives \& constraints" tab, which is enabled after inserting the data for each case, showing the organs of interest (e.g target), as well as the organs at risk. Here, the dose optimization can be configured by defining multiple, typical planning objectives and constraints to achieve the optimal dose distribution in different structures or organs.

Last tab is the "visualization" tab, where the user can set visualization properties. For instance, there is a "slice selection" scroll bar, to select and display a specific $2 \mathrm{D}$ image out of the $3 \mathrm{D}$ voxelized patient volume. In addition, there is the "plane selection" button where the user can switch between axial, sagittal and coronal view. In addition, the "visualize plan/beam lines" button is important, which one can enable for a better understanding of the different beam angles of the irradiation plan.

During the PTMC tutorials, first the TG-119 is demonstrated after the description of important medical physics terms that are also provided in the PTMC webpage. The TG-119 is an acrylic sample that is also known as C-phantom, due to its characteristic shape (Figure 5).

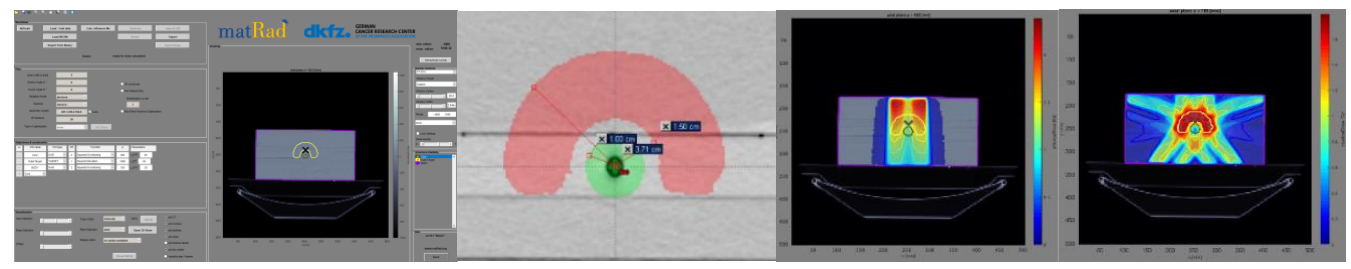

Figure 5: matRad GUI; TG-119 sample; TG-119 irradiation with a single angle of photons and five angles.

This phantom is usually used by professionals for quality assurance to verify the proper function of their equipment. In this case, the aim is to avoid the central bar, which represents the Organ At Risk (OAR, organs more sensitive to irradiation), and irradiate only the C-shape, which is the target where the cancer tumour is located. For this tutorial, two photon treatment plans were designed utilizing a single beam angle and five beam angles to demonstrate characteristics of intensity modulated photon treatment plans. This example demonstrates how the number of beam angles influences the resulting dose distribution. The results can be seen in Figure 5, where the red zones are the areas irradiated the most and the blue ones the least.

Then, for a real-life application, the liver case is typically being demonstrated. In this case, the complexity of the treatment plan is increased due to the sensitive areas that should be taken into consideration during planning. Three radiation methods were used, photons, protons, and carbons. For the photon treatment plan, five different angles were used, focused on the side where the tumor is located. For the proton and carbon treatment plans, a single angle was used. The results can be seen in Figure 6. 

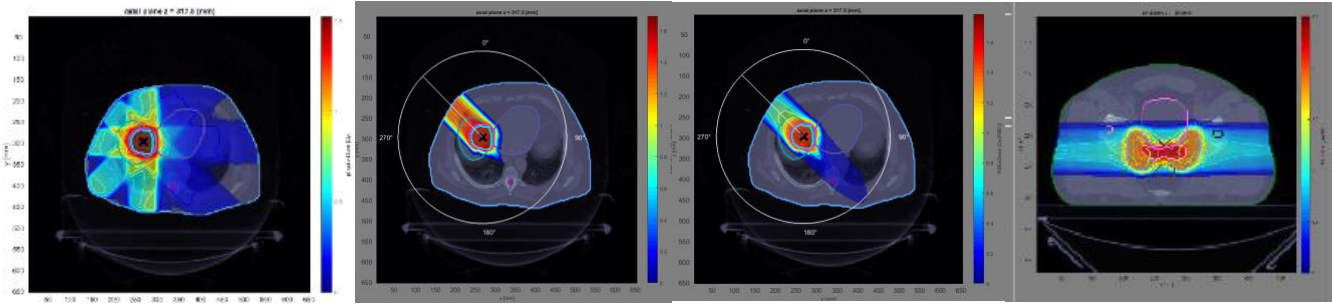

Figure 6: (left to right) Liver case treatment plan with photons, with protons and with carbons. Prostate treatment plan with carbons.

The matRad software also provides Dose-Volume Histograms (DVH) that show how much dose is received by each volume in percent. Ideally, $100 \%$ of the prescribed dose should be received by the target structure while $100 \%$ of the organ at risk should receive zero dose. DVHs are very useful, especially when comparing quantitatively the effects of the different radiation methods. An example of a DVH can be seen Figure 7, for the liver case.
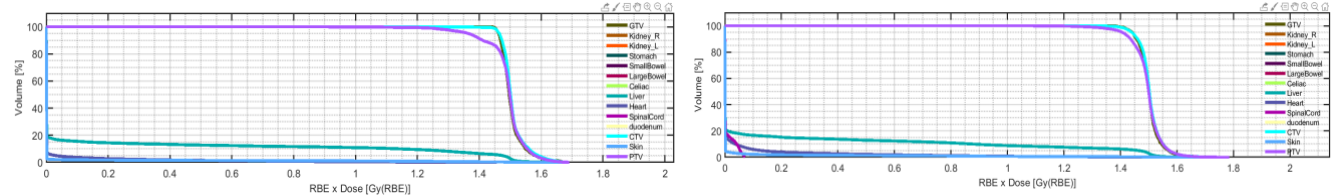

Figure 7: DVH for the liver case with protons (left) and carbons (right).

Through the graphical interpretations of the DVH, the importance of the carbon and proton irradiation is highlighted, since a better and more accurate dose deposition is achieved in the region of importance, avoiding unwanted irradiation to the healthy tissue, thanks to their Bragg peak characteristics and increased radiobiological effectiveness.

The aim of the tutorial is also to demonstrate that there is no a priori "correct" method for treating patients, but the radiation physicist, in collaboration with the medical doctor, must define an individual patient-tailored treatment plan for each case, which in some cases might be the combination of two radiation methods or of different treatment techniques. During the hands-on treatment planning process, the advantages of particle therapy become apparent for the most successful treatment of the patient.

\section{Heavy Ion Therapy MasterClass School}

Motivated by the received feedback, the format of the PTMC and its pedagogical elements were employed for the fullweek Heavy Ion Therapy MasterClass (HITM) school [17], which was organised within the framework of the HITRIplus [19] EU-funded project, from 17-21 May 2021, online, with the support of the PTMC core team of volunteers (Figure 4). It was attended by over 1000 participants, from all over the world, that ranged from undergraduate students to Masters, $\mathrm{PhDs}$, early stage researchers but also professional practitioners, and involved a total of 36 speakers. Participants greatly appreciated the multidisciplinary approach of the school and the presentations from top experts that started from basics and covered most recent developments and future plans in heavy-ion therapy. Recordings are available via the HITM school web page [17].

The worldwide reach of the HITM school demonstrates the increasing interest in heavy-ion therapy but also the power of networks; the participation to the IMC and the HITM school (maps of Figure 8) match almost one-to-one, since the school was also announced through the IPPOG network.
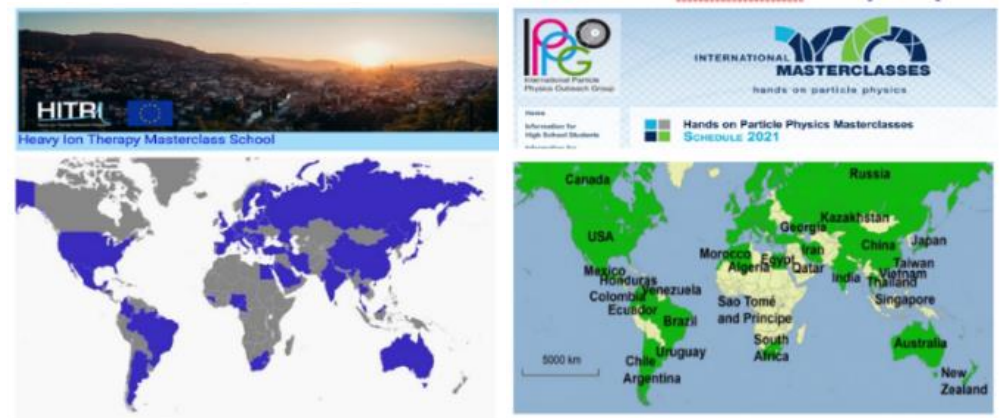

Figure 8: World-wide reach of HITM school and IMC. 
An IPPOG colleague from Benha University in Egypt contributed with an interesting initiative by organising the participation of some 100 students as part of the University curriculum, who joined the sessions from an auditorium, and ensuring the support and presence of the dean for the opening session. Figure 4, shows the discussion of their PTMC results for a liver tumour case. Starting from the first pilot in 2019, addressing school children (Figure 9), the PTMC is now adapted to address also university students and has expanded its reach to a new generation of scientists.

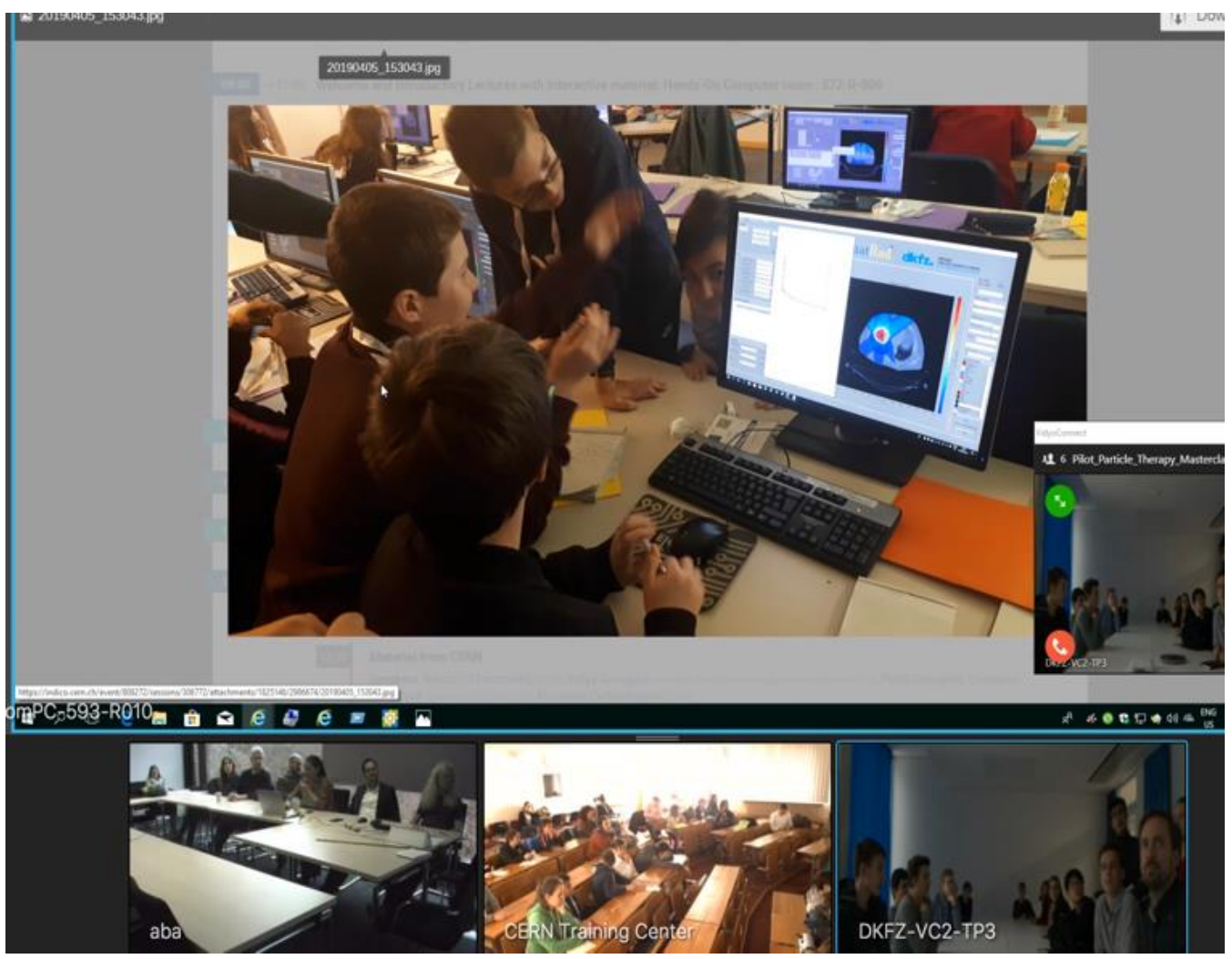

Figure 9: First PTMC pilot in 2019.

\section{Summary}

The PTMC addressing high-school students and the HITM school addressing university students make the young generation aware of the various possibilities that physics, and STEM studies in general, open up for career opportunities in emerging fields where often there is lack of specialised personnel. They also enhance awareness about modern methods for cancer therapy and new research avenues, that are expanding world-wide; and where clearly the development of technology and expertise of research laboratories is important. Participants are impressed performing a treatment planning like professionals and motivated to contribute to the further advancements of this multidisciplinary field, that opens new horizons. Overall, the opportunities for properly trained scientists is a take-home message.

They also come to appreciate the power of networks and importance of international collaborations working on common goals but also the different challenges and approaches of different disciplines (starting from the relative characterisation of "heavy" ions by physicians and physicists) and, hence, the importance of finding common grounds. The PTMC also highlights the importance of close collaboration among research institutes and their coordinated contributions to this multidisciplinary field. It is not a coincidence that the PTMC was developed with the support of GSI, where carbon-ion therapy was pioneered in Europe, CERN, where the PIMMS designs for ion-therapy machines emerged, and DKFZ, that pushes the frontiers of cancer research.

The motivation for the development of the PTMC after the development of the ALICE MasterClasses [18, 21], was to spread the message that heavy-ions and the tools developed for fundamental research, can also be used for cancer therapy. Several institutes choose to perform them back-to-back which enhances the effect. For the developers and all contributors, institute organisers, tutors, moderators, is a great satisfaction to see the impact and experience the feedback of students at all levels; from the dedication of the core team volunteers, the engagement of assistants, to the interest of 
high-school students. With clear statements they spell out the strong impact it has on them the realisation that what they learn can have tangile impact to society and health.

\section{Acknowledgements}

We further thank Mark Bangert for his aid in the initial local test at GSI as well as the Heidelberg Life Science Lab and Kathrin Platzer for supporting the DKFZ masterclasses; the ENLIGHT [20] coordinator, Manjit Dosanjh, for her guidance, introducing the relevant network, and providing material; the students of the Uni of Sarajevo UNSA, and Uni of Thessaloniki AUTH, that contributed setting up the PTMC web pages, dedicated trainings, material in local languages etc; the students of UNAM, Mexico, for setting up the "PTMC in a kit" first in Spanish and then in English; ALICE, GSI, $\mathrm{CNAO}$ colleagues for being eager to offer real-time online visits and moderate video-conferences. The help and support of all colleagues that contributed to this endeavour and the taking off of the PTMC should be acknowledged; also, the dedication of the core team of volunteers from AUTH and UNSA for keeping up with the work and challenges that came with the growing interest for the PTMC. Last but not least, the ARIES EU-funded project and the private "Three Physicists Foundation" for supporting the PTMC development and dissemination.

\section{References}

1. IMC : https://physicsmasterclasses.org/publications: https://physicsmasterclasses.org/index.php?cat=papers

2. IPPOG webpage: https://ippog.org

3. Particle Therapy MasterClass webpage: https://indico.cern.ch/event/840212/

4. GSI: https://www.gsi.de/start/aktuelles

5. CERN: https://home.cern/

6. DKFZ: https://www.dkfz.de/en/index.html

7. Bryant, Philip J, PIMMS : Proton-ion medical machine study, in CERN Yellow Reports: Monographs, ebook: 10.5170/CERN-2000-006

8. HIT: https://www.klinikum.uni-heidelberg.de/interdisziplinaere-zentren/heidelberger-ionenstrahl-therapiezentrumhit/

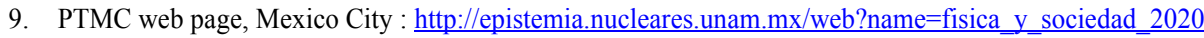

PTMC webpage, Puebla : https://www.fcfm.buap.mx/ParticulasElementales/seminarios/PT/index.html

10. ALICE collaboration : https://alice-collaboration.web.cern.ch/

11. CNAO: https://fondazionecnao.it/en/

12. matRad - an open-source dose calculation and treatment planning toolkit, https://github.com/e0404/matRad https://doi.org/10.5281/zenodo.3879615

13. Cisternas E., Mairani A., Ziegenhein P., Jäkel O., Bangert M. (2015) matRad - a multi-modality open-source 3D treatment planning toolkit. In: Jaffray D. (eds) World Congress on Medical Physics and Biomedical Engineering, June 7-12, 2015, Toronto, Canada. IFMBE Proceedings, vol 51. Springer, Cham. https://doi.org/10.1007/978-3-319$\underline{19387-8 \quad 391}$

14. Wieser, H.-P., Cisternas, E., Wahl, N., Ulrich, S., Stadler, A., Mescher, H., Müller, L.-R., Klinge, T., Gabrys, H., Burigo, L., Mairani, A., Ecker, S., Ackermann, B., Ellerbrock, M., Parodi, K., Jäkel, O. and Bangert, M. (2017), Development of the open-source dose calculation and optimization toolkit matRad. Med. Phys., 44: 2556-2568. https://doi.org/10.1002/mp.122

15. D. Craft, M. Bangert, T. Long, D. Papp, J. Unkelbach, Shared data for intensity modulated radiation therapy (IMRT) optimization research: the CORT dataset, GigaScience, Volume 3, Issue 1, December 2014, 2047-217X-3-37, https://doi.org/10.1186/2047-217X-3-37

16. Medical Dictionary: https://medical-dictionary.thefreedictionary.com/bixel

17. HITM school webpage: https://indico.cern.ch/event/1019104/

18. L. Graczykowski, P. Nowakowski, P. Foka, New developments for ALICE MasterClasses and the new Particle Therapy MasterClass, in Proceedings of CHEP 2019,

[2005.02215] New developments for ALICE MasterClasses and the new Particle Therapy MasterClass (arxiv.org)

19. Hitriplus : https://www.hitriplus.eu/

20. ENLIGHT : https://enlight.web.cern.ch/

21. Y. Foka, M. Janik, ALICE Masterclass on strangeness, in EPJ Web of Conferences, Volume 71, 2014,

https://www.epj-

conferences.org/articles/epjconf/abs/2014/08/epjconf_icnfp2013 00057/epjconf_icnfp2013 00057.html 